\title{
Identification of biomarkers of sugar sweetened beverage intake
}

\author{
H. Gibbons, B. A. McNulty, A. P. Nugent, M. J. Gibney and L. Brennan \\ UCD Institute of Food and Health, University College Dublin, Belfield, Dublin 4, Ireland
}

High consumption of sugar sweetened beverages (SSB) has been associated with risk of type 2 diabetes ${ }^{(1)}$ and weight gain ${ }^{(2)}$. In order to enhance our understanding of these links between SSB and disease, reliable and accurate dietary assessment methods of a person's food intake are essential. As current dietary assessment methods are associated with a number of random and systematic errors, focus has shifted to biomarkers of dietary intake. The identification of novel biomarkers of SSB intake, through the application of metabolomics, offers the potential of a more objective measure of SSB intake. This study aims to identify a panel of biomarkers indicative of SSB intake from a large cohort study and subsequently validate this in an acute intervention study.

Dietary intake data and ${ }^{1} \mathrm{H}$ nuclear magnetic resonance $\left({ }^{1} \mathrm{H}\right.$ NMR) urine spectra from 565 participants of the National Adult Nutrition Survey (NANS) ${ }^{(3)}$ were used for this analysis. Dietary intake data was obtained from 4-day food diaries and this was reduced into 34 food groups. Heat map analysis was performed to identify correlations between ${ }^{1} \mathrm{H}$ NMR spectral regions and SSB intakes. Positively correlated peaks within the spectral regions were then compared between low and high consumers of SSB intake. Metabolites were identified and quantified using Chenomx Profiler (Version 7.5, Chenomx Inc.; Edmonton, Canada). Receiver operating characteristic (ROC) analysis was performed to assess sensitivity and specificity of biomarkers. Validation of this panel of biomarkers involved an acute intervention study. 10 participants provided a fasting first-void urine sample and urine samples at 2,4 and 6 hours following the consumption of a SSB drink. A 24 hour urine sample was also collected. Following ${ }^{1} \mathrm{H}$ NMR spectroscopic profiling of the urine, multivariate data analysis was applied.

Heat map analysis identified SSBs as having strong correlations with a number of spectral regions. Spectral peaks which were positively correlated with SSBs and significantly increased in the high consumers $(83-1155 \mathrm{~g})$ were identified asformate, citrulline, taurine and isocitrate. All 4 metabolites were significantly increased in high consumers $(\mathrm{P}<0.05)$. This panel of biomarkers had an area under the curve (AUC) of 0.8 for ROC analysis and a sensitivity and specificity of 0.7 and 0.8 respectively. Following acute consumption of a SSB drink all 4 metabolites increased in the urine, validating there use as potential biomarkers. Future work will ascertain how to translate this panel of markers for use in nutrition epidemiology.

This analysis was supported by NutriTech (Project Number 289511).

1. The InterAct consortium (2013) Diabetologia 56, 1520-1530

2. DeBoer MD, Scharf RJ, Demmer RT (2013) Pediactrics 132, 1-8

3. Irish Universities Nutrition Alliance (2012) The National Adult Nutrition Survey. http://www.iuna.net/ 